Homology, Homotopy and Applications, vol.14(2), 2012, pp.129-142

\title{
WEIGHT STRUCTURE ON KONTSEVICH'S NONCOMMUTATIVE MIXED MOTIVES
}

\author{
GONÇALO TABUADA
}

\author{
(communicated by Claude Cibils)
}

\begin{abstract}
In this article we endow Kontsevich's triangulated category $\mathrm{KMM}_{k}$ of noncommutative mixed motives with a nondegenerate weight structure in the sense of Bondarko. As an application we obtain: (1) a convergent weight spectral sequence for every additive invariant (e.g., algebraic $K$-theory, cyclic homology, topological Hochschild homology, etc.); (2) a ring isomorphism between $K_{0}\left(\mathrm{KMM}_{k}\right)$ and the Grothendieck ring of the category of noncommutative Chow motives; (3) a precise relationship between Voevodsky's (virtual) mixed motives and Kontsevich's noncommutative (virtual) mixed motives.
\end{abstract}

\section{Weight structure}

In his seminal talk [11], Kontsevich introduced the triangulated category $\mathrm{KMM}_{k}$ of noncommutative mixed motives (over a base commutative ring $k$ ) and conjectured the existence of a "different" $t$-structure on this category. In this article we formalize Kontsevich's beautiful insight and illustrate some of its important consequences. Recall from $[\mathbf{1 4}, \mathbf{1 7}, \mathbf{1 8}]$ the construction of the additive category NChow $_{k}$ of noncommutative Chow motives. Our formalization of the "different" $t$-structure is the following:

Theorem 1.1. There exist two full subcategories $\mathrm{KMM}_{k}^{w \geqslant 0}$ and $\mathrm{KMM}_{k}^{w \leqslant 0}$ of $\mathrm{KMM}_{k}$ verifying the following seven conditions:

(i) $\mathrm{KMM}_{k}^{w \geqslant 0}$ and $\mathrm{KMM}_{k}^{w \leqslant 0}$ are additive and idempotent complete.

(ii) $\mathrm{KMM}_{k}^{w \geqslant 0} \subset \mathrm{KMM}_{k}^{w \geqslant 0}[1]$ and $\mathrm{KMM}_{k}^{w \leqslant 0}[1] \subset \mathrm{KMM}_{k}^{w \leqslant 0}$.

(iii) For every $M \in \mathrm{KMM}_{k}^{w \geqslant 0}$ and $N \in \mathrm{KMM}_{k}^{w \leqslant 0}[1]$ we have $\operatorname{Hom}_{\mathrm{KMM}_{k}}(M, N)=0$.

(iv) For every $M \in \mathrm{KMM}_{k}$ there is a distinguished triangle

$$
\begin{aligned}
& \qquad N_{2}[-1] \longrightarrow M \longrightarrow N_{1} \longrightarrow N_{2} \\
& \text { with } N_{1} \in \mathrm{KMM}_{k}^{w \leqslant 0} \text { and } N_{2} \in \mathrm{KMM}_{k}^{w \geqslant 0} \text {. }
\end{aligned}
$$

The author was partially supported by the NEC Award-2742738 and by the FCT-Portugal grants PTDC/MAT/098317/2008 and SFRH/BSAB/1116/2011.

Received April 10, 2012, revised June 24, 2012; published on November 19, 2012.

2000 Mathematics Subject Classification: 14A22, 18D20, 18G40, 19L10.

Key words and phrases: weight structure, weight spectral sequence, Grothendieck ring, Picard group, Voevodsky motive, Kontsevich noncommutative motive.

Article available at http://intlpress.com/HHA/v14/n2/a8 and doi:10.4310/HHA.2012.v14.n2.a8

Copyright (C) 2012, International Press. Permission to copy for private use granted. 
(v) $\mathrm{KMM}_{k}=\cup_{l \in \mathbb{Z}} \mathrm{KMM}_{k}^{w \geqslant 0}[-l]=\cup_{l \in \mathbb{Z}} \mathrm{KMM}_{k}^{w \leqslant 0}[-l]$.

(vi) There is a natural equivalence of categories $\mathrm{NChow}_{k} \simeq \mathrm{KMM}_{k}^{w \geqslant 0} \cap \mathrm{KMM}_{k}^{w \leqslant 0}$.

(vii) $\cap_{l \in \mathbb{Z}} \mathrm{KMM}_{k}^{w \geqslant 0}[-l]=\cap_{l \in \mathbb{Z}} \mathrm{KMM}_{k}^{w \leqslant 0}[-l]=\{0\}$.

Items (i)-(iv) assert that the triangulated category $\mathrm{KMM}_{k}$ is endowed with a weight structure $w$ (also known in the literature $[\mathbf{1 3}]$ as a co-t-structure) in the sense of Bondarko [6, Def. 1.1.1]. Item (v) asserts that $w$ is bounded, item (vi) that the heart of $w$ can be identified with the category of noncommutative Chow motives, and item (vii) that $w$ is non-degenerate. Theorem 1.1 should then be regarded as the noncommutative analogue of the Chow weight structure on Voevodsky's triangulated category of mixed motives; see $[\mathbf{6}, \S \S 6.5-6.6]$.

\section{Acknowledgements}

The author is very grateful to Paul Balmer, Mikhail Bondarko, Yuri Manin and Greg Stevenson for useful discussions and motivating questions, as well as to the anonymous referee for his comments.

\section{Weight spectral sequences}

Let dgcat $_{k}$ be the category of (small) dg categories over a fixed base commutative ring $k$; consult Keller's ICM address [10].

Definition 2.1. Let $L(-): \operatorname{dgcat}_{k} \rightarrow \mathcal{M}$ be a functor with values in a symmetric monoidal stable model category; see $[\mathbf{9}, \S 4$ and $\S 7]$. We say that $L$ is an additive invariant if it verifies the following three conditions:

(i) Filtered colimits are mapped to filtered colimits.

(ii) Derived Morita equivalences (i.e., dg functors which induce an equivalence on the associated derived categories; see $[\mathbf{1 0}, \S 4.6])$ are mapped to weak equivalences.

(iii) Split exact sequences (i.e., sequences of dg categories which become split exact after passage to the associated derived categories; see $[\mathbf{1 5}, \S 13])$ are mapped to direct sums

$$
0 \longrightarrow \mathcal{A} \leftrightarrows \mathcal{B} \leftrightarrows \mathcal{C} \longrightarrow 0 \mapsto L(\mathcal{A}) \oplus L(\mathcal{C}) \simeq L(\mathcal{B})
$$

in the homotopy category $\mathrm{Ho}(\mathcal{M})$.

Proposition 2.2. Every additive invariant $L(-)$ gives rise to a triangulated functor $L(-): \mathrm{KMM}_{k} \longrightarrow \mathrm{Ho}(\mathcal{M})$; which we still denote by $L(-)$.

Consider the following compositions:

$$
L_{n}(-): \mathrm{KMM}_{k} \stackrel{L(-)}{\longrightarrow} \mathrm{Ho}(\mathcal{M}) \stackrel{\operatorname{Hom}(\mathbf{1}[n],-)}{\longrightarrow} \mathrm{Ab} \quad n \in \mathbb{Z},
$$

where 1 stands for the $\otimes$-unit of $\mathcal{M}$ and $A b$ for the category of abelian groups.

Example 2.3 (Algebraic $K$-theory). Recall from [10, $\S 5.2]$ that the (connective) algebraic $K$-theory functor $K(-):$ dgcat $_{k} \rightarrow \mathrm{Sp}$, with values in the category of spectra, satisfies the above conditions (i)-(iii) and hence is an additive invariant. 
Example 2.4 (Hochschild and cyclic homology). Recall from $[\mathbf{1 0}, \S 5.3]$ that the Hochschild and cyclic homology functors $H H(-), H C(-)$ : dgcat ${ }_{k} \rightarrow \mathcal{C}(k)$, with values in the category of complexes of $k$-modules, are additive invariants. In these examples the associated functors $H H_{n}(-)$ and $H C_{n}(-)$ take values in the abelian category of $k$-modules.

Example 2.5 (Negative cyclic homology). Recall from [7, Example 7.10] that the mixed complex functor $C(-): \operatorname{dgcat}_{k} \rightarrow \mathcal{C}(\Lambda)$, with values in the category of mixed complexes, satisfies the above conditions (i)-(iii) and hence is an additive invariant. Moreover, as explained in [7, Example 8.10], the associated functors $C_{n}(-)$ agree with the negative cyclic homology functors $H C_{n}^{-}(-)$.

Example 2.6 (Periodic cyclic homology). Periodic cyclic homology is not an additive invariant since its definition uses infinite products, and these do not commute with filtered colimits. Nevertheless, it factors through $\mathrm{KMM}_{k}$ as follows: recall from [7, Example 7.11] that we have a 2-perioditization functor $P(-): \mathcal{C}(\Lambda) \rightarrow k[u]$-Comod, with values in the category of comodules over the Hopf algebra $k[u]$. This functor preserves weak equivalences and hence by applying the above Proposition 2.2 to $C(-)$ we obtain the following composed triangulated functor:

$$
\mathrm{KMM}_{k} \stackrel{C(-)}{\longrightarrow} \mathrm{Ho}(\mathcal{C}(\Lambda)) \stackrel{P(-)}{\longrightarrow} \mathrm{Ho}(k[u] \text {-Comod }) .
$$

As explained in [7, Example 8.11], the associated functors $((P \circ C)(-))_{n}$ agree with the periodic cyclic homology functors $H P_{n}(-)$.

Example 2.7 (Topological Hochschild homology). Recall from [3] that the topological Hochschild homology functor $T H H(-):$ dgcat $_{k} \rightarrow$ Sp is also an example of an additive invariant.

Recall from [16, Prop. 2.5] and [19, Thm. 2.8] the construction of the following natural transformations between additive invariants:

$$
\begin{aligned}
t r & : K(-) \\
c h^{2 i}: K(-) & \Rightarrow H C(-)[-2 i], \\
c h^{-}: K(-) & \Rightarrow C(-) .
\end{aligned}
$$

By first evaluating these natural transformations at a noncommutative mixed motive $M$, and then passing to the associated functors (1) we obtain, respectively, the Dennis trace maps, the higher Chern characters, and the negative Chern characters:

$$
\begin{aligned}
t r_{n}: K_{n}(M) & \rightarrow H H_{n}(M), \\
c h_{n}^{2 i}: K_{n}(M) & \rightarrow H C_{n+2 i}(M), \\
c h_{n}^{-}: K_{n}(M) & \rightarrow H C_{n}^{-}(M) .
\end{aligned}
$$

Theorem 2.8. Under the preceding notations the following holds:

(i) To every noncommutative mixed motive $M$ we can associate a cochain (weight) complex of noncommutative Chow motives

$$
t(M): \cdots \longrightarrow M^{(i-1)} \longrightarrow M^{(i)} \longrightarrow M^{(i+1)} \longrightarrow \cdots .
$$


Moreover, the assignment $M \mapsto t(M)$ gives rise to a conservative functor from $\mathrm{KMM}_{k}$ towards a certain weak category of complexes $K_{\mathfrak{m}}\left(\right.$ NChow $\left._{k}\right)$; see [6, $\S 3.1]$.

(ii) Every additive invariant $L(-)$ yields a convergent (weight) spectral sequence

$$
E_{1}^{p q}(M)=L_{-q}\left(M^{(p)}\right) \Rightarrow L_{-p-q}(M) .
$$

Moreover, (3) is functorial on $M$ after the $E_{1}$-term.

(iii) The above natural transformations (2) respect the spectral sequence (3).

Intuitively speaking, item (i) shows us that all the information concerning a noncommutative mixed motive can be encoded into a cochain complex. Items (ii) and (iii) endow the realm of noncommutative mixed motives with a new powerful computational tool which is, moreover, well-behaved with respect to the classical Chern characters. We intend to develop this computational aspect in future work.

\section{Grothendieck ring and Picard group}

As explained in $[\mathbf{1 4}, \mathbf{1 7}]$, the categories $\mathrm{KMM}_{k}$ and $\mathrm{NChow}_{k}$ are endowed with a symmetric monoidal structure induced by the tensor product of dg categories. Hence, the Grothendieck group of $\mathrm{KMM}_{k}$ (considered as a triangulated category) and the Grothendieck group of $\mathrm{NChow}_{k}$ (considered as an additive category) are endowed with a ring structure. The symmetric monoidal structure allows us also to consider the Picard groups $\operatorname{Pic}\left(\mathrm{NChow}_{k}\right)$ and $\operatorname{Pic}\left(\mathrm{KMM}_{k}\right)$, i.e., the multiplicative groups of (isomorphism classes of) $\otimes$-invertible objects. As explained in [20], every Azumaya $k$-algebra is $\otimes$-invertible in the homotopy category of dg categories. Since these $k$-algebras remain $\otimes$-invertible in $\mathrm{NChow}_{k}$, they furnish us with a large class of examples of elements of $\mathrm{Pic}\left(\mathrm{NChow}_{k}\right)$.

Theorem 3.1. The equivalence of categories of item (vi) of Theorem 1.1 gives rise:

(i) to a ring isomorphism $K_{0}\left(\mathrm{NChow}_{k}\right) \stackrel{\sim}{\rightarrow} K_{0}\left(\mathrm{KMM}_{k}\right)$,

(ii) to an injective homomorphism $\operatorname{Pic}\left(\mathrm{NChow}_{k}\right) \times \mathbb{Z} \rightarrow \operatorname{Pic}\left(\mathrm{KMM}_{k}\right)$.

Informally speaking, item (i) shows us that "up to extension" the categories $\mathrm{KMM}_{k}$ and $\mathrm{NChow}_{k}$ have the same isomorphism classes. In what concerns item (ii), the factor $\mathbb{Z}$ corresponds to shifting.

\section{Voevodsky's versus Kontsevich's mixed motives}

In this section we assume that $k$ is a perfect field. By working with rational coefficients the weight structure of Theorem 1.1 allows us to establish a precise relationship between Voevodsky's (virtual) mixed motives and Kontsevich's noncommutative (virtual) mixed motives.

Recall from $[\mathbf{2 1}, \S 4]$ the construction of the triangulated category $\operatorname{DM}_{g m}(k)_{\mathbb{Q}}$ of mixed motives (with rational coefficients) and of the functor $M_{g m}(-): \operatorname{Sm}_{k} \rightarrow$ $\mathrm{DM}_{g m}(k)_{F}$ that associates to every smooth $k$-scheme $X$ its geometric motive $M_{g m}(X)$. On the other hand, let $\operatorname{NChow}(k)_{\mathbb{Q}}$ and $\operatorname{KMM}(k)_{\mathbb{Q}}$ be the categories obtained from NChow $_{k}$ and $\mathrm{KMM}_{k}$ by first tensoring each abelian group of morphisms with $\mathbb{Q}$ and 
then passing to the idempotent completion. The triangulated categories $\mathrm{DM}_{g m}(k)_{\mathbb{Q}}$ and $\operatorname{KMM}(k)_{\mathbb{Q}}$ are endowed with a symmetric monoidal structure, and so the associated Grothendieck groups carry an induced ring structure.

Theorem 4.1. There exists a zig-zag of triangulated functors

$$
\operatorname{DM}_{g m}(k)_{\mathbb{Q}} \stackrel{r}{\rightarrow} K^{b}\left(\mathrm{NChow}(k)_{\mathbb{Q}}\right) \stackrel{t}{\leftarrow} \mathrm{KMM}(k)_{\mathbb{Q}},
$$

where $K^{b}(-)$ stands for the homotopy category of bounded complexes. The functor $t$ is conservative (i.e., it reflects isomorphisms), and for every smooth projective $k$-scheme $X$ we have a natural isomorphism $r\left(M_{g m}(X)_{\mathbb{Q}}\right) \simeq t\left(\mathcal{D}_{\text {perf }}^{\mathrm{dg}}(X)\right)$, where $\mathcal{D}_{\text {perf }}^{\mathrm{dg}}(X)$ stands for the (unique) $d g$ enhancement of the derived category of perfect complexes of $\mathcal{O}_{X^{-}}$ modules; see [12]. Moreover, the functor $r$ induces a well-defined ring homomorphism

$$
K_{0}\left(\mathrm{DM}_{g m}(k)_{\mathbb{Q}}\right)\left[[\mathbb{Q}(-1)[2]]^{-1}\right] \longrightarrow K_{0}\left(\operatorname{KMM}(k)_{\mathbb{Q}}\right),
$$

where $[\mathbb{Q}(-1)[2]]$ denotes the class of the Tate motive.

Informally speaking, Theorem 4.1 establishes a "bridge" between the commutative and the noncommutative world. All the information concerning Voevodsky's mixed motives versus Kontsevich's noncommutative mixed motives can now be compared using the above zig-zag (4). For instance, the Tate motive $\mathbb{Q}(-1)[2]$ is virtually $\otimes$-trivial from the noncommutative viewpoint. We believe that this "bridge" opens new horizons and opportunities of research by enabling the interchange of results, techniques, ideas, and insights between the commutative and the noncommutative world.

\section{Proofs}

\subsection{Proof of Theorem 1.1}

Recall from [11] that a dg category $\mathcal{A}$ is called smooth if it is perfect as a bimodule over itself and proper if for each ordered pair of objects $(x, y)$ in $\mathcal{A}$, the complex of $k$-modules $\mathcal{A}(x, y)$ is perfect. Recall also that Kontsevich's construction of $\mathrm{KMM}_{k}$ decomposes in three steps:

(1) First, consider the category $\mathrm{KPM}_{k}$ (enriched over spectra) whose objects are the smooth and proper $\mathrm{dg}$ categories, whose morphisms from $\mathcal{A}$ to $\mathcal{B}$ are given by the (connective) algebraic $K$-theory spectrum $K\left(\mathcal{A}^{\mathrm{op}} \otimes^{\mathbb{L}} \mathcal{B}\right)$, and whose composition is induced by the (derived) tensor product of bimodules.

(2) Then, take the formal triangulated envelope of $\mathrm{KPM}_{k}$. Objects in this new category are formal finite extensions of formal shifts of objects in $\mathrm{KPM}_{k}$. Let $\mathrm{KTM}_{k}$ be the associated homotopy category.

(3) Finally, pass to the pseudo-abelian envelope of $\mathrm{KTM}_{k}$. The resulting category $\mathrm{KMM}_{k}$ is what Kontsevich named the category of noncommutative mixed motives.

Recall from $[\mathbf{1 5}, \S 15]$ the construction of the additive motivator of dg categories $\operatorname{Mot}_{\mathrm{dg}}^{\text {add }}$ and the associated base triangulated category $\operatorname{Mot}_{\mathrm{dg}}^{\text {add }}(e)$. The analogue 
of [7, Prop. 8.5] (with the above definition ${ }^{1}$ of $\mathrm{KMM}_{k}$ and $\operatorname{Mot}_{\mathrm{dg}}^{\mathrm{loc}}(e)$ replaced by $\left.\operatorname{Mot}_{\mathrm{dg}}^{\text {add }}(e)\right)$ holds similarly. Hence, $\mathrm{KMM}_{k}$ can be identified with the smallest thick triangulated subcategory of $\operatorname{Mot}_{\mathrm{dg}}^{\text {add }}(e)$ spanned by the noncommutative mixed motives of smooth and proper dg categories. Similarly, $\mathrm{KTM}_{k}$ can be identified with the smallest triangulated subcategory of $\operatorname{Mot}_{\mathrm{dg}}^{\text {add }}(e)$ spanned by the noncommutative mixed motives of smooth and proper dg categories. In what follows, we will assume that these identifications have been made.

Now, recall from $[\mathbf{1 4}, \mathbf{1 7}, \mathbf{1 8}]$ that the category NChow $_{k}$ of noncommutative Chow motives is defined as the pseudo-abelian envelope of the category whose objects are the smooth and proper $\mathrm{dg}$ categories, whose morphisms from $\mathcal{A}$ to $\mathcal{B}$ are given by the Grothendieck group $K_{0}\left(\mathcal{A}^{\mathrm{oP}} \otimes^{\mathbb{L}} \mathcal{B}\right)$, and whose composition is induced by the (derived) tensor product of bimodules.

Proposition 5.1. There is a natural fully-faithful functor

$$
\Phi: \mathrm{NChow}_{k} \longrightarrow \mathrm{KMM}_{k}
$$

Proof. Given dg categories $\mathcal{A}$ and $\mathcal{B}$, let $\mathcal{D}\left(\mathcal{A}^{\text {op }} \otimes^{\mathbb{L}} \mathcal{B}\right)$ be derived category of $\mathcal{A}-\mathcal{B}$ bimodules and $\operatorname{rep}(\mathcal{A}, \mathcal{B}) \subset \mathcal{D}\left(\mathcal{A}^{\text {op }} \otimes^{\mathbb{L}} \mathcal{B}\right)$ the full triangulated subcategory spanned by those $\mathcal{A}$ - $\mathcal{B}$-bimodules $X$ such that for every object $x \in \mathcal{A}$, the associated $\mathcal{B}$-module $X(x,-)$ is perfect; consult $[\mathbf{1 0}, \S 4.2]$ for further details. Recall from $[\mathbf{1 4}, \S 5]$ the construction of the additive category $\mathrm{Hmo}_{0}$ : the objects are the $\mathrm{dg}$ categories, the morphisms from $\mathcal{A}$ to $\mathcal{B}$ are given by the Grothendieck group $K_{0} \operatorname{rep}(\mathcal{A}, \mathcal{B})$ of the triangulated category $\operatorname{rep}(\mathcal{A}, \mathcal{B})$, and the composition is induced by the (derived) tensor product of bimodules. There is a natural functor

$$
\mathcal{U}_{\mathrm{A}}: \text { dgcat }_{k} \longrightarrow \mathrm{Hmo}_{0}
$$

which sends a $\operatorname{dg}$ functor $F: \mathcal{A} \rightarrow \mathcal{B}$ to the class in $K_{0} \operatorname{rep}(\mathcal{A}, \mathcal{B})$ of the corresponding $\mathcal{A}$ - $\mathcal{B}$-bimodule. On the other hand, recall from $[\mathbf{1 5}, \S 15]$ the construction of the functor

$$
\mathcal{U}_{a}: \operatorname{dgcat}_{k} \longrightarrow \operatorname{Mot}_{\mathrm{dg}}^{\text {add }}(e) .
$$

As proved in [14, Thms. 4.6 and 6.3], $\mathcal{U}_{\mathrm{A}}$ is the universal functor with values in an additive category which inverts derived Morita equivalences and sends split exact sequences $^{2}$ to direct sums (see condition (iii) of Definition 2.1). Since these conditions are satisfied by the functor $\mathcal{U}_{a}$ (see $\left[\mathbf{1 5}\right.$, Thm. 15.4]) and $\operatorname{Mot}_{\mathrm{dg}}^{\text {add }}(e)$ is an additive category (since it is triangulated), we obtain an induced additive functor $\Psi$ making the following diagram commute:

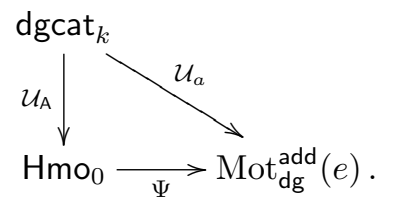

\footnotetext{
${ }^{1}$ In $[7, \S 8.2]$ we have considered non-connective algebraic $K$-theory since we were interested in the relationship with secondary $K$-theory. However, Kontsevich's original definition is in terms of connective algebraic $K$-theory.

${ }^{2}$ This condition can equivalently be formulated in terms of a general semi-orthogonal decomposition in the sense of Bondal-Orlov; see [14, Thm. 6.3(4)].
} 
Let us denote by $\mathrm{Hmo}_{0}^{\mathrm{sp}} \subset \mathrm{Hmo}_{0}$ the full subcategory of smooth and proper dg categories. When $\mathcal{A}$ (and $\mathcal{B}$ ) is smooth and proper we have a natural isomorphism

$$
\operatorname{Hom}_{\mathrm{Hmo}_{0}^{\mathrm{sp}}}(\mathcal{A}, \mathcal{B}):=K_{0} \operatorname{rep}(\mathcal{A}, \mathcal{B}) \simeq K_{0}\left(\mathcal{A}^{\mathrm{op}} \otimes^{\mathbb{L}} \mathcal{B}\right) ;
$$

see $\left[\mathbf{7}\right.$, Lemma 4.9]. Hence, we observe that the category NChow $_{k}$ of noncommutative Chow motives is the pseudo-abelian envelope of $\mathrm{Hmo}_{0}^{\text {sp }}$. Since by construction the triangulated category $\mathrm{KMM}_{k} \subset \operatorname{Mot}_{\mathrm{dg}}^{\text {add }}(e)$ is idempotent complete, the composition $\mathrm{Hmo}_{0}^{\text {sp }} \subset \mathrm{Hmo}_{0} \stackrel{\Psi}{\longrightarrow} \mathrm{Mot}_{\mathrm{dg}}^{\text {add }}(e)$ extends then to a well-defined additive functor

$$
\Phi: \mathrm{NChow}_{k} \longrightarrow \mathrm{KMM}_{k} \subset \operatorname{Mot}_{\mathrm{dg}}^{\mathrm{add}}(e) .
$$

Finally, the fact that $\Phi$ is fully-faithful follows from the following computation:

$$
\operatorname{Hom}_{\operatorname{Mot}_{\mathrm{dg}}^{\text {add }}(e)}\left(\mathcal{U}_{a}(\mathcal{A}), \mathcal{U}_{a}(\mathcal{B})\right) \simeq K_{0} \operatorname{rep}(\mathcal{A}, \mathcal{B}) \simeq K_{0}\left(\mathcal{A}^{\mathrm{op}} \otimes^{\mathbb{L}} \mathcal{B}\right)
$$

for every smooth and proper dg category $\mathcal{A}$; see [15, Prop. 16.1].

In order to prove Theorem 1.1, let us now verify the conditions of Bondarko's [6, Thm. 4.3.2 II], with $\underline{C}$ the triangulated category $\mathrm{KTM}_{k} \subset \mathrm{KMM}_{k}$ and $H$ the essential image of the composition $\mathrm{Hmo}_{0}^{\text {sp }} \subset \mathrm{NChow}_{k} \rightarrow \mathrm{KMM}_{k}$. By construction, $H$ generates $\mathrm{KTM}_{k}$ in the sense of [6, page 11]. Moreover, given any two smooth and proper dg categories $\mathcal{A}$ and $\mathcal{B}$, we have the following computation:

$$
\operatorname{Hom}_{\mathrm{KMM}_{k}}(\Phi(\mathcal{A}), \Phi(\mathcal{B})[-n]) \simeq \begin{cases}K_{n}\left(\mathcal{A}^{\mathrm{op}} \otimes^{\mathbb{L}} \mathcal{B}\right) & n \geqslant 0 \\ 0 & n<0\end{cases}
$$

This follows from [15, Prop. 16.1] combined with the specific construction of $\Phi$. Hence, $H \subset \mathrm{KTM}_{k}$ is negative in the sense of [6, Def. 4.3.1(1)]. The conditions of [6, Thm. 4.3.2 II] are then satisfied, and so we conclude that there exists a unique bounded weight structure $w$ on $\mathrm{KTM}_{k}$ whose heart is the pseudo-abelian envelope of $H$. Note that the heart is then equivalent to NChow $_{k}$ under the above fully-faithful functor (6). Since the weight structure $w$ is bounded, [6, Prop. 5.2.2] implies that $w$ can be extended from $\mathrm{KTM}_{k}$ to $\mathrm{KMM}_{k}$. The heart remains exactly the same since the category NChow $_{k}$ is by construction idempotent complete. By [6, Defs. 1.1.1 and 1.2.1] we then conclude that conditions (i)-(vi) of Theorem 1.1 are verified, where $\mathrm{KMM}_{k}^{w \geqslant 0}$ (resp. $\mathrm{KMM}_{k}^{\leqslant 0}$ ) is the smallest idempotent complete and extension-stable subcategory of $\mathrm{KMM}_{k}$ (see [6, Def. 1.3.1]) containing the objects $\Phi\left(\mathrm{NChow}_{k}\right)[n]$, $n \leqslant 0$ (resp. $\left.\Phi\left(\mathrm{NChow}_{k}\right)[n], n \geqslant 0\right)$. It remains then to verify condition (vii). We start by proving the equality $\cap_{l \in \mathbb{Z}} \mathrm{KMM}_{k}^{w \geqslant 0}[-l]=\{0\}$.

Proposition 5.2. For every noncommutative mixed motive $M$, there exists an integer $j \in \mathbb{Z}$ (which depends on $M$ ) such that for every $N \in \Phi\left(\right.$ NChow $\left._{k}\right)$ we have

$$
\operatorname{Hom}_{\mathrm{KMM}_{k}}(N, M[i])=0 \text { when } i>j .
$$

Proof. Let $\mathcal{C}$ be a full subcategory of $\operatorname{Mot}_{\mathrm{dg}}^{\text {add }}(e)$ containing the zero object. Let us denote by $\mathcal{C}[\mathbb{Z}]$ the category $\cup_{n \in \mathbb{Z}} \mathcal{C}[n]$, by $\mathcal{C}^{\natural}$ the idempotent completion of $\mathcal{C}$ inside $\operatorname{Mot}_{\mathrm{dg}}^{\text {add }}(e)$, and by $\operatorname{Ext}(\mathcal{C})$ the subcategory of $\operatorname{Mot}_{\mathrm{dg}}^{\text {add }}(e)$ formed by the objects $\mathcal{O}$ for 
which there exists a distinguished triangle

$$
M_{1} \longrightarrow \mathcal{O} \longrightarrow M_{2} \longrightarrow M_{1}[1]
$$

with $M_{1}$ and $M_{2}$ in $\mathcal{C}$. Note that $\mathcal{C} \subseteq \operatorname{Ext}(\mathcal{C})$. Consider the following

Vanishing condition. There exists an integer $j \in \mathbb{Z}$ such that, for every object $N \in$ $\Phi\left(\right.$ NChow $\left._{k}\right)$, we have

$$
\operatorname{Hom}_{\operatorname{Mot}_{\mathrm{dg}}^{\text {add }}(e)}(N, \mathcal{O}[i])=0 \quad \text { when } i>j .
$$

We now show that if by hypothesis the above vanishing condition holds for every object $\mathcal{O}$ of $\mathcal{C}$, then it holds also for every object of the following categories:

(1) The category $\mathcal{C}[\mathbb{Z}]$ : This is the case since every object in $\mathcal{C}[\mathbb{Z}]$ is of the form $\mathcal{O}[n]$, with $n$ and integer and $\mathcal{O} \in \mathcal{C}$.

(2) The category $\operatorname{Ext}(\mathcal{C})$ : By construction every object $\mathcal{O}$ of $\operatorname{Ext}(\mathcal{C})$ fits in the above distinguished triangle (11). Let $j_{1}$ and $j_{2}$ be the integers of the vanishing condition which are associated to $M_{1}$ and $M_{2}$, respectively. Then, by choosing $j:=\max \left\{j_{1}, j_{2}\right\}$ we observe that the object $\mathcal{O}$ also verifies the above vanishing condition.

(3) The category $\mathcal{C}^{\natural}$ : This is the case since every object in $\mathcal{C}^{\natural}$ is a direct summand of an object in $\mathcal{C}$; recall that $\operatorname{Mot}_{\mathrm{dg}}^{\text {add }}(e)$ admits arbitrary sums and so every idempotent splits.

Let us now apply the above general arguments to the category $\mathcal{C}=\Phi\left(\mathrm{NChow}_{k}\right)$. By computation (9) the above vanishing condition holds for every object (with $j=$ $0)$. Recall that $\mathrm{KMM}_{k}$ is the smallest thick triangulated subcategory of $\operatorname{Mot}_{\mathrm{dg}}^{\text {add }}(e)$ spanned by the objects $N \in \Phi\left(\right.$ NChow $\left._{k}\right)$. Hence, every object $M \in \mathrm{KMM}_{k}$ belongs to the category obtained from $\Phi\left(\mathrm{NChow}_{k}\right)$ by applying the above constructions (1)-(3) a finite number of times (the number of times depends on $M$ ). As a consequence, we conclude that $M$ satisfies the above vanishing condition and so the proof is finished.

Let $M \in \cap_{l \in \mathbb{Z}} \operatorname{KMM}_{k}^{w \geqslant 0}[-l]$. Note that equality (10) can be re-written as

$$
\operatorname{Hom}_{\mathrm{KMM}_{k}}(N[-i], M)=0 \text { when } i>j \text {. }
$$

Since $\mathrm{KMM}_{k}^{w \geqslant 0}$ is the smallest idempotent complete and extension stable subcategory of $\mathrm{KMM}_{k}$ containing the objects $\Phi\left(\mathrm{NChow}_{k}\right)[n], n \leqslant 0$, we conclude from (12) that $\operatorname{Hom}_{\mathrm{KMM}_{k}}(\mathcal{O}, M)=0$ for every object $\mathcal{O}$ belonging to $\mathrm{KMM}_{k}^{w \geqslant 0}[-l]$ with $l>j$. Since by hypothesis $M \in \mathrm{KMM}_{k}^{w \geqslant 0}[-l]$, we then conclude, by the Yoneda lemma, that $M=0$ in $\mathrm{KMM}_{k}^{w \geqslant 0}[-l]$ (with $l>j$ ) and hence in $\cap_{l \in \mathbb{Z}} \mathrm{KMM}_{k}^{w \geqslant 0}[-l]$. Let us now prove the equality $\cap_{l \in \mathbb{Z}} \mathrm{KMM}_{k}^{w \leqslant 0}[-l]=\{0\}$.

Proposition 5.3. For every non-trivial noncommutative mixed motive $M$, there exists an integer $j \in \mathbb{Z}$, an object $N \in \Phi\left(\mathrm{NChow}_{k}\right)$, and a non-trivial morphism $f: N[j] \rightarrow M$.

Proof. We prove the following equivalent statement: if $\operatorname{Hom}_{\mathrm{KMM}_{k}}(N[n], M)=0$ for every integer $n \in \mathbb{Z}$ and object $N \in \Phi\left(\right.$ NChow $\left._{k}\right)$, then $M=0$. Recall that $\mathrm{KMM}_{k}$ 
is the smallest thick triangulated subcategory of $\operatorname{Mot}_{\mathrm{dg}}^{\mathrm{add}}(e)$ spanned by the objects $N \in \Phi\left(\right.$ NChow $\left._{k}\right)$. The class of objects $\mathcal{O}$ in $\operatorname{Mot}_{\mathrm{dg}}^{\text {add }}(e)$ satisfying the equalities

$$
\operatorname{Hom}_{\operatorname{Mot}_{\mathrm{dg}}^{\text {add }}(e)}(\mathcal{O}[n], M)=0 \quad n \in \mathbb{Z}
$$

is clearly stable under extensions and direct factors. Since by hypothesis it contains the objects $N \in \Phi\left(\mathrm{NChow}_{k}\right)$ it also contains all the objects of the category $\mathrm{KMM}_{k}$. Hence, by taking $\mathcal{O}=M$ and $n=0$, the identity morphism of $M$ allows us to conclude that $M=0$.

Let $M \in \cap_{l \in \mathbb{Z}} \mathrm{KMM}_{k}^{w \leqslant 0}[-l]$. If by hypothesis $M$ is non-trivial, then the morphism $f$ of Proposition 5.3 gives rise to to a non-trivial morphism

$$
0 \neq f[-j]: N \longrightarrow M[-j] .
$$

Since by construction $N$ belongs to $\mathrm{KMM}_{k}^{w \geqslant 0}$, condition (iii) of Theorem 1.1 combined with the non-trivial morphism (13) implies that $M[-j] \notin \mathrm{KMM}_{k}^{w \leqslant 0}[1]$. Hence, $M \notin$ $\mathrm{KMM}_{k}^{w \leqslant 0}[1+j]$, and so we obtain a contradiction with our hypothesis. This allows us to conclude that $M=0$, and so the proof of Theorem 1.1 is finished.

\subsection{Proof of Proposition 2.2}

The category dgcat $_{k}$ carries a (cofibrantly generated) Quillen model structure whose weak equivalences are the derived Morita equivalences; see [14, Thm. 5.3]. Hence, it gives rise to a well-defined Grothendieck derivator $\mathrm{HO}\left(\right.$ dgcat $\left._{k}\right)$; consult [7, Appendix A] for the notion of Grothendieck derivator. Since by hypothesis $\mathcal{M}$ is stable and $L(-)$ satisfies conditions (i)-(iii) of Definition 2.1, we then obtain a well-defined additive invariant of $\mathrm{dg}$ categories $\mathrm{HO}\left(\right.$ dgcat $\left._{k}\right) \rightarrow \mathrm{HO}(\mathcal{M})$ in the sense of $[\mathbf{1 5}$, Notation 15.5]. By the universal property of [15, Thm. 15.4], this additive invariant factors through Mot $_{d g}^{\text {add }}$ giving rise to a homotopy colimit preserving morphism of derivators $\operatorname{Mot}_{\mathrm{dg}}^{\mathrm{add}} \rightarrow \mathrm{HO}(\mathcal{M})$ and hence to a triangulated functor $\operatorname{Mot}_{\mathrm{dg}}^{\mathrm{add}}(e) \rightarrow \mathrm{Ho}(\mathcal{M})$ on the underlying base categories. As explained in the proof of Theorem 1.1, the category $\mathrm{KMM}_{k}$ can be identified with a full triangulated subcategory of $\operatorname{Mot}_{\mathrm{dg}}^{\text {add }}(e)$. The composition obtained

$$
L(-): \mathrm{KMM}_{k} \subset \operatorname{Mot}_{\mathrm{dg}}^{\mathrm{add}}(e) \longrightarrow \mathrm{Ho}(\mathcal{M})
$$

is then the triangulated functor mentioned in Proposition 2.2.

\subsection{Proof of Theorem 2.8}

As explained in the proof of Theorem 1.1, the category $\mathrm{KMM}_{k}$ is endowed with a non-degenerate bounded weight structure $w$ whose heart is equivalent to the category NChow $_{k}$ of noncommutative Chow motives. In particular, we have the following equalities:

$$
\mathrm{KMM}_{k}^{+}=\mathrm{KMM}_{k}=\mathrm{KMM}_{k}^{-} \text {; }
$$

see [6, Def. 1.3.5]. Hence, by combining [6, Thm. 3.2.2 II] with [6, Thm. 3.3.1] one obtains the claim of item (i). By Proposition 2.2 every additive invariant $L(-)$ gives rise to a triangulated functor $L(-): \mathrm{KMM}_{k} \rightarrow \mathrm{Ho}(\mathcal{M})$ and hence to a composed 
functor

$$
\mathrm{KMM}_{k} \stackrel{L(-)}{\longrightarrow} \mathrm{Ho}(\mathcal{M}) \stackrel{\operatorname{Hom}(\mathbf{1},-)}{\longrightarrow} \mathrm{Ab} .
$$

Note that (14) is homological, i.e., it sends distinguished triangles to long exact sequences, and that we have the following identifications:

$$
\operatorname{Hom}(\mathbf{1}, L(M[-i])) \simeq \operatorname{Hom}(\mathbf{1}, L(M)[-i]) \simeq \operatorname{Hom}(\mathbf{1}[i], L(M))=L_{i}(M) .
$$

Since the weight structure $w$ is bounded, we have $\mathrm{KMM}_{k}=\mathrm{KMM}_{k}^{b}$; see [6, Def. 1.3.5]. Hence, item (ii) follows from [6, Thms. 2.3.2 II and IV] (with $H=(14)$ ) and from the above identifications (15). Finally, item (iii) follows from [6, Thm. 2.3.2 III] since all the Chern characters (2) are natural transformations of additive invariants.

Remark 5.4. As the above proof clearly shows, Theorem 2.8(ii) applies also to periodic cyclic homology; see Example 2.6.

\subsection{Proof of Theorem 3.1}

Let us start by proving item (i). As explained in [7, Thm. 7.5], the functor (8) is symmetric monoidal. Since (7) is also symmetric monoidal, we conclude from the construction of (6) that this latter functor is also symmetric monoidal. Recall from the proof of Theorem 1.1 that the category $\mathrm{KMM}_{k}$ is endowed with a bounded weight structure $w$. Since the functor (6) is symmetric monoidal, the heart $\Phi\left(\mathrm{NChow}_{k}\right)$ of $w$ is then a full additive symmetric monoidal subcategory of $\mathrm{KMM}_{k}$. Hence, by combining [6, Thm. 5.3.1] with [6, Remark 5.3.2], one obtains the ring isomorphism $K_{0}\left(\mathrm{NChow}_{k}\right) \simeq K_{0}\left(\mathrm{KMM}_{k}\right)$.

Let us now prove item (ii), i.e., that the homomorphism

$$
\operatorname{Pic}\left(\mathrm{NChow}_{k}\right) \times \mathbb{Z} \longrightarrow \operatorname{Pic}\left(\mathrm{KMM}_{k}\right) \quad(\mathcal{A}, n) \mapsto \Phi(\mathcal{A})[n]
$$

is injective. As explained above, the functor $\Phi$ is symmetric monoidal and so the homomorphism (16) is well-defined. Given non-isomorphic objects $\mathcal{A}$ and $\mathcal{B}$ of NChow , one needs to show that the objects $\Phi(\mathcal{A})[n]$ and $\Phi(\mathcal{B})[m]$ of $\mathrm{KMM}_{k}$ remain nonisomorphic for all $n, m \in \mathbb{Z}$. When $n=m$ this follows from the fact that the functor $\Phi$ is fully-faithful; see Proposition 5.1. When $n>m$ this follows from the natural isomorphisms

$$
\operatorname{Hom}_{\mathrm{KMM}_{k}}(\Phi(\mathcal{A})[n], \Phi(\mathcal{B})[m]) \simeq \operatorname{Hom}_{\mathrm{KMM}_{k}}(\Phi(\mathcal{A}), \Phi(\mathcal{B})[n-m]) \stackrel{(9)}{\simeq} 0 .
$$

Similarly, when $n<m$ this follows from the natural isomorphisms

$$
\operatorname{Hom}_{\mathrm{KMM}_{k}}(\Phi(\mathcal{B})[m], \Phi(\mathcal{A})[n]) \simeq \operatorname{Hom}_{\mathrm{KMM}_{k}}(\Phi(\mathcal{B}), \Phi(\mathcal{A})[m-n]) \stackrel{(9)}{\simeq} 0 .
$$

\subsection{Proof of Theorem 4.1}

By construction, the category $\operatorname{NChow}(k)_{\mathbb{Q}}$ is additive, symmetric monoidal and, moreover, idempotent complete. In what concerns $\operatorname{KMM}(k)_{\mathbb{Q}}$ recall from (9) that $\operatorname{Hom}_{\mathrm{KMM}_{k}}(\Phi(k), \Phi(k)) \simeq K_{0}(k)$. Since by hypothesis $k$ is a field, the endomorphism ring of the $\otimes$-unit $\Phi(k)$ is $K_{0}(k)=\mathbb{Z}$. Hence, by first applying [1, Thm. 3.6] to the multiplicative set $S:=\mathbb{Z} \backslash\{0\} \subset K_{0}(k)$ and then using [2, Thm. 1.5], one obtains a canonical $\otimes$-triangulated structure on $\operatorname{KMM}(k)_{\mathbb{Q}}$. Now, recall from $[\mathbf{1 7}$, Thm. 1.1] 
and $[\mathbf{1 8}$, Thm. 4.6] the following construction:

$$
\mathrm{Chow}(k)_{\mathbb{Q}} \stackrel{\pi}{\rightarrow} \operatorname{Chow}(k)_{\mathbb{Q}} /-\otimes \mathbb{Q}(1) \stackrel{R}{\rightarrow} \mathrm{NChow}(k)_{\mathbb{Q}} .
$$

Some explanations are in order: $\operatorname{Chow}(k)_{\mathbb{Q}}$ stands for the category of Chow motives (with rational coefficients) and $\operatorname{Chow}(k)_{\mathbb{Q}} /-\otimes \mathbb{Q}(1)$ stands for the orbit category associated to the Tate motive $\mathbb{Q}(1)$. Roughly speaking, two Chow motives which differ from a Tate twist become isomorphic in Chow $(k)_{\mathbb{Q}} /-\otimes \mathbb{Q}(1)$. The functors $\pi$ and $R$ are additive, $\mathbb{Q}$-linear, conservative, symmetric monoidal, and $R$ is, moreover, fully-faithful. They give then rise to triangulated functors

$$
K^{b}\left(\operatorname{Chow}(k)_{\mathbb{Q}}\right) \longrightarrow K^{b}\left(\operatorname{Chow}(k)_{\mathbb{Q}} /-\otimes \mathbb{Q}(1)\right) \longrightarrow K^{b}\left(\operatorname{NChow}(k)_{\mathbb{Q}}\right)
$$

between the homotopy categories of bounded complexes. Recall from $[\mathbf{6}, \S \S 6.5-6.6]$ that since by hypothesis $k$ is a perfect field the triangulated category $\operatorname{DM}_{g m}(k)_{\mathbb{Q}}$ carries a bounded Chow weight structure. Its heart is the essential image of a fullyfaithful functor ${ }^{3}$

$$
\operatorname{Chow}(k)_{\mathbb{Q}} \longrightarrow \operatorname{DM}_{g m}(k)_{\mathbb{Q}}
$$

sending $\mathbb{Q}(1)$ to $\mathbb{Q}(-1)[2]$. By $[6, \S 5]$ there exists then a well-defined conservative triangulated (weight complex) functor $t_{0}: \operatorname{DM}_{g m}(k)_{\mathbb{Q}} \rightarrow K^{b}\left(\operatorname{Chow}(k)_{\mathbb{Q}}\right)$. By precomposing (18) with $t_{0}$ we obtain then the functor $r$ of the zig-zag (4).

Let us now show that the triangulated category $\operatorname{KMM}(k)_{\mathbb{Q}}$ carries a bounded weight structure, as in Theorem 1.1, with heart naturally isomorphic to Chow $(k)_{\mathbb{Q}}$. By construction, (6) gives rise to a fully-faithful functor $\Phi_{\mathbb{Q}}: \operatorname{NChow}(k)_{\mathbb{Q}} \rightarrow \operatorname{KMM}(k)_{\mathbb{Q}}$. Moreover, since $\mathrm{NChow}_{k}$ generates $\mathrm{KMM}_{k}$, the additive category $\mathrm{NChow}(k)_{\mathbb{Q}}$ generates $\operatorname{KMM}(k)_{\mathbb{Q}}$. Furthermore, the computation (9) implies that

$$
\operatorname{Hom}_{\mathrm{KMM}(k)_{\mathbb{Q}}}\left(\Phi_{\mathbb{Q}}(\mathcal{A}), \Phi_{\mathbb{Q}}(\mathcal{B})[-n]\right) \simeq \begin{cases}K_{n}\left(\mathcal{A}^{\mathrm{op}} \otimes^{\mathbb{L}} \mathcal{B}\right)_{\mathbb{Q}} & n \geqslant 0 \\ 0 & n<0\end{cases}
$$

for all smooth and proper dg categories $\mathcal{A}$ and $\mathcal{B}$. Hence, one concludes (as in the proof of Theorem 1.1) that the conditions (i)-(vi) are verified. In what concerns condition (vii), the equality $\cap_{l \in \mathbb{Z}} \operatorname{KMM}(k)_{\mathbb{Q}}^{w \geqslant 0}[-l]=\{0\}$ follows from the vanishing condition

$$
\operatorname{Hom}_{\mathrm{KMM}(k)_{\mathbb{Q}}}(N, M[i])=0 \text { when } i>j ;
$$

see Proposition 5.2. In what concerns the equality $\cap_{l \in \mathbb{Z}} \operatorname{KMM}(k)_{\mathbb{Q}}^{w \leqslant 0}[-l]=\{0\}$, the proof is similar to the one of Proposition 5.3 (and of what it follows).

Now, let us endow the triangulated category $\operatorname{KMM}(k)_{\mathbb{Q}}$ with a negative differential graded enhancement $\operatorname{KMM}(k)_{\mathbb{Q}}^{\mathrm{dg}}$. Given a smooth and proper dg category $\mathcal{C}$, let us write $\widehat{\mathcal{C}}$ for the associated Waldhausen category [22] of perfect $\mathcal{C}$-modules, with weak equivalences the quasi-isomorphisms and cofibrations the morphisms which admit retractions as morphisms of graded $\mathcal{C}$-modules; see [10, §5.2]. Following GilletSoulé $[\mathbf{8}, \S 6.2]$, we denote by $M_{*}(\widehat{\mathcal{C}})$ the negative differential graded cochain complex of $\mathbb{Q}$-vector spaces computing rational algebraic $K$-theory, i.e., such that $H^{-n} M_{*}(\widehat{\mathcal{C}}) \simeq$ $K_{n}(\mathcal{C})_{\mathbb{Q}}$ for $n \geqslant 0$. This data allows the following construction:

\footnotetext{
${ }^{3}$ Denoted by $R$ in loc. cit.
} 
(1) First, consider the category $\operatorname{KPM}(k)_{\mathbb{Q}}^{\mathrm{dg}}$ (enriched over negative differential graded cochain complexes) whose objects are the smooth and proper dg categories, whose morphisms from $\mathcal{A}$ to $\mathcal{B}$ are given by the cochain complex $M_{*}\left(\widehat{\mathcal{A}^{\mathrm{op}} \otimes^{\mathbb{L}}} \mathcal{B}\right)$, and whose composition is induced by the (derived) tensor product of bimodules;

(2) Then, take Bondal-Kapranov's pretriangulated envelope $\operatorname{Tr}^{+}\left(\operatorname{KPM}(k)_{\mathbb{Q}}\right)$ of the $\operatorname{dg}$ category $\operatorname{KPM}(k)_{\mathbb{Q}}^{\mathrm{dg}}$; consult [4] for details.

(3) Finally, pass to the pseudo-abelian envelope $\operatorname{KMM}(k)_{\mathbb{Q}}^{\mathrm{dg}}$ of $\operatorname{Tr}^{+}\left(\operatorname{KPM}(k)_{\mathbb{Q}}\right)$.

By the above computation (20) and the description of $\mathrm{KMM}_{k}$ given at the beginning of the proof of Theorem 1.1 one observes that $\operatorname{KMM}(k)_{\mathbb{Q}}^{\mathrm{dg}}$ enhances $\operatorname{KMM}(k)_{\mathbb{Q}}$. As explained in $[\mathbf{6}, \S 6]$, we then obtain a well-defined conservative triangulated (weight complex) functor $t: \operatorname{KMM}(k)_{\mathbb{Q}} \rightarrow K^{b}\left(\mathrm{NChow}(k)_{\mathbb{Q}}\right)$. This concludes the construction of the zig-zag relating $\operatorname{DM}_{g m}(k)_{\mathbb{Q}}$ with $\operatorname{KMM}(k)_{\mathbb{Q}}$.

Let us now prove the natural isomorphism $r\left(M_{g m}(X)_{\mathbb{Q}}\right) \simeq t\left(\mathcal{D}_{\text {perf }}^{\mathrm{dg}}(X)\right)$ for every smooth projective $k$-scheme $X$. Recall from [17, Thm. 1.1] the construction of the following commutative diagram:

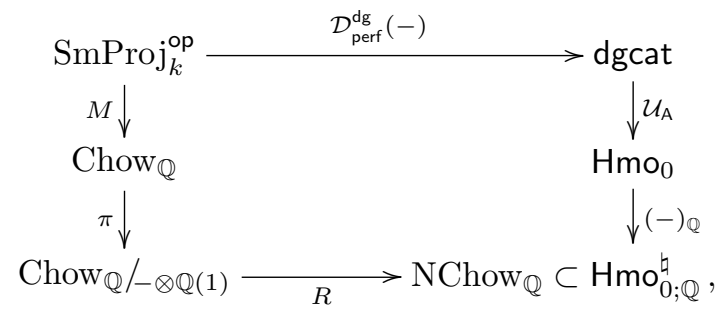

where $\mathrm{SmProj}_{k}$ stands for the category of smooth projective $k$-schemes. Since by hypothesis $X$ is projective the mixed motive $M_{g m}(X)_{\mathbb{Q}}$ belongs to the essential image of the functor (19), i.e., it belongs to the heart of the Chow weight structure on $\mathrm{DM}_{g m}(X)_{\mathbb{Q}}$. By $\left[\mathbf{6}\right.$, Thm. 3.3.1 IV] the bounded complex $t_{0}\left(M_{g m}(X)_{\mathbb{Q}}\right)$ can then be identified with $M(X)$ (considered as a complex concentrated in degree zero). Hence, by definition of $r$, one obtains a natural isomorphism $r\left(M_{g m}(X)_{\mathbb{Q}}\right) \simeq(R \circ \pi \circ M)(X)$ of complexes in $K^{b}\left(\mathrm{NChow}(k)_{\mathbb{Q}}\right)$ concentrated in degree zero. By the above commutative diagram $(21)$, one concludes that $(R \circ \pi \circ M)(X)$ also identifies with the noncommutative motive $\mathcal{U}_{\mathrm{A}}\left(\mathcal{D}_{\text {perf }}^{\mathrm{dg}}(X)_{\mathbb{Q}}\right)$, which we will simply denote by $\mathcal{D}_{\text {perf }}^{\mathrm{dg}}(X)$. Since $\mathcal{D}_{\text {perf }}^{\text {dg }}(X)$ clearly belongs to the heart of the weight structure on $\operatorname{KMM}(k)_{\mathbb{Q}}$ constructed above, one concludes from $\left[6\right.$, Thm. 3.3.1 IV] that $t\left(\mathcal{D}_{\text {perf }}^{\mathrm{dg}}(X)\right) \simeq \mathcal{D}_{\text {perf }}^{\mathrm{dg}}(X)$. By combining the above arguments, one then obtains a natural isomorphism $r\left(M_{g m}(X)_{\mathbb{Q}}\right) \simeq$ $t\left(\mathcal{D}_{\text {perf }}^{\mathrm{dg}}(X)\right)$ for every smooth projective $k$-scheme $X$.

Let us now describe the ring homomorphism (5). As explained in the proof of Theorem 3.1(i), the fully-faithful functor $\Phi_{\mathbb{Q}}: \operatorname{NChow}(k)_{\mathbb{Q}} \rightarrow \operatorname{KMM}(k)_{\mathbb{Q}}$ induces a ring homomorphism $K_{0}\left(\operatorname{NChow}(k)_{\mathbb{Q}}\right) \simeq K_{0}\left(\operatorname{KMM}(k)_{\mathbb{Q}}\right)$. Similarly, as explained in [5, Corollary 6.4.3], the fully-faithful functor (19) induces a ring isomorphism between $K_{0}\left(\right.$ Chow $\left.(k)_{\mathbb{Q}}\right)$ and $K_{0}\left(\operatorname{DM}_{g m}(k)_{\mathbb{Q}}\right)$. Hence, since the functors $\pi$ and $R$ are additive, $\mathbb{Q}$-linear, and symmetric monoidal, the above composition (17) gives rise to a welldefined ring homomorphism

$$
K_{0}\left(\mathrm{DM}_{g m}(k)_{\mathbb{Q}}\right) \longrightarrow K_{0}\left(\mathrm{KMM}(k)_{\mathbb{Q}}\right) .
$$


WEIGHT STRUCTURE ON KONTSEVICH'S NONCOMMUTATIVE MIXED MOTIVES 14

By construction the class $[\mathbb{Q}(-1)[2]] \in K_{0}\left(\mathrm{DM}_{g m}(k)_{\mathbb{Q}}\right)$ is first mapped to $[\mathbb{Q}(1)] \in$ $K_{0}\left(\operatorname{Chow}(k)_{\mathbb{Q}}\right)$ and then to the class of the $\otimes$-unit of Chow $(k)_{\mathbb{Q}} /-\otimes \mathbb{Q}(1)$. As a consequence, (22) gives rise to the ring homomorphism (5).

\section{References}

[1] P. Balmer, Spectra, spectra, spectra - Tensor triangular spectra versus Zariski spectra of endomorphism rings, Algebr. Geom. Topol. 10 (2010), no. 3, 15211563.

[2] P. Balmer and M. Schlichting, Idempotent completion of triangulated categories, J. Algebra 236 (2001), no. 2, 819-834.

[3] A. Blumberg and M. Mandell, Localization theorems in topological Hochschild homology and topological cyclic homology, Geom. \& Top. 16 (2012), 10511120.

[4] A. Bondal and M. Kapranov, Framed triangulated categories (Russian) Mat. Sb. 181 (1990), no. 5, 669-683; translation in Math. USSR-Sb. 70 (1991), no. 1, 93-107.

[5] M. V. Bondarko, Differential graded motives: weight complex, weight filtrations and spectral sequences for realizations; Voevodsky versus Hanamura, J. of the Inst. of Math. of Jussieu 8 (2009), no. 1, 39-97.

[6] M. V. Bondarko, Weight structures vs. $t$-structures; weight filtrations, spectral sequences, and complexes (for motives and in general), Journal of K-Theory 6 (2010), no. 3, 387-504.

[7] D.-C. Cisinski and G. Tabuada, Symmetric monoidal structure on Noncommutative motives, Journal of K-theory 9 (2012), no. 2, 201-268.

[8] H. Gillet and C. Soulé, Motivic weight complexes for arithmetic varieties, J. Algebra 322 (2009), no. 9, 3088-3141.

[9] M. Hovey, Model Categories, Mathematical Surveys and Monographs 63, American Mathematical Society, Providence, RI, 1999.

[10] B. Keller, On differential graded categories, International Congress of Mathematicians (Madrid), Vol. II, 151-190, Eur. Math. Soc., Zürich (2006).

[11] M. Kontsevich, Noncommutative motives, Talk at the Institute for Advanced Study on the occasion of the $61^{\text {st }}$ birthday of Pierre Deligne, October 2005, Video available at http://video.ias.edu/Geometry-and-Arithmetic.

[12] V. Lunts and D. Orlov, Uniqueness of enhancement for triangulated categories, J. Amer. Math. Soc. 23 (2010), no. 3, 853-908.

[13] D. Pauksztello, Compact corigid objects in triangulated categories and co-tstructures, Cent. Eur. J. Math. 6 (2008), no. 1, 25-42.

[14] G. Tabuada, Invariants additifs de dg-catégories, Int. Math. Res. Not. 53 (2005), 3309-3339.

[15] G. Tabuada, Higher $K$-theory via universal invariants, Duke Math. J. 145 (2008), no. 1, 121-206.

[16] G. Tabuada, A simple criterion for extending natural transformations to higher K-theory, Documenta Mathematica 16 (2011), 657-668. 
[17] G. Tabuada, Chow motives versus noncommutative motives, Available at arXiv:1103.0200, to appear in Journal of Noncommutative Geometry.

[18] G. Tabuada, A guided tour through the garden of noncommutative motives, to appear in Clay Mathematics Proceedings, Volume 16 (2013).

[19] G. Tabuada, Products, multiplicative Chern characters, and finite coefficients via non-commutative motives, Available at arXiv:1101.0731.

[20] B. Toën, Derived Azumaya algebras and generators for twisted derived categories, Available at arXiv:1002.2599.

[21] V. Voevodsky, Triangulated categories of motives over a field, in Cycles, transfers, and motivic homology theories, Annals of Mathematics Studies 143, 188238, Princeton University Press, Princeton, NJ, 2000.

[22] F. Waldhausen, Algebraic $K$-theory of spaces, in Algebraic and geometric topology (New Brunswick, NJ, 1983), 318-419, Lecture Notes in Math. 1126, Springer-Verlag, New York (1985).

Gonçalo Tabuada tabuada@math.mit.edu

Department of Mathematics, MIT, Cambridge, MA 02139 and Departamento de Matematica, FCT-UNL, Quinta da Torre, 2829-516, Caparica, Portugal 